\title{
APLICACIÓN DE TÉCNICAS EXPERIMENTALES PARA DETERMINACIÓN DE FALLAS EN JUNTAS POR TORNILLO
}

* Msc. Profesor Titular de la Universidad del Valle

\author{
Jaime Sánchez R. * \\ Adolfo León Gómez P.* \\ Argemiro Collazos P. *
}

\section{RESUMEN}

En todas las ramas de la Ingeniería, desde los primeros tiempos hasta el presente y en el futuro, existen dos métodos fundamentales para resolver problemas que surgen en el descubrimiento del conocimiento y su aplicación a las necesidades de la sociedad: a. Modelamiento Teórico. b. Medición Experimental.

En la Ingeniería esto es cierto independientemente de la disciplina (Ingeniería Mecánica, Ingeniería Eléctrica, Ingeniería Química, etc.) o la función de la Ingeniería 
(Diseño, manufactura, etc.). Mientras que algunos problemas son adecuadamente tratados usando sólo teoría, o solamente experimentación, la mayoría requieren una juiciosa combinación de estas técnicas. Esta es la situación que se presenta en este estudio, donde además de efectuar el análisis teórico del problema se emplean técnicas experimentales para recoger información que nos permita determinar la causa de fallas en juntas por tornillo.

\section{ABSTRACT}

In all branches of engineering, from the earliest times to the present and into the future, there exist only two fundamental approaches to solve problems that arise in the discovery of knowledge and its application to society's needs: a. Theoretical modeling. b. Experimental measurement

In engineering, this is true regardless of the discipline (mechanical engineering, electrical engineering, chemical engineering, etc.) or the engineering function (design, manufacturing, etc.). While some problems are adequately treated by using only theory, or only experimentation, most require judiciously chosen mix of these techniques. This is the situation that is presented in this paper, where besides to realize the theoretical analysis of the problem are used experimental measurements to obtain information that allow us to determine the cause of failure in the threaded fasteners of an assembled joint.

\section{INTRODUCCIÓN}

Por solicitud de EPSA, el Grupo de Investigación en Mejoramiento Industrial GIMI de la actual Escuela de Ingeniería Mecánica de la Facultad de Ingeniería de la Universidad del Valle realizó el análisis teórico y experimental para determinar las causas de las fallas de las uniones por tornillo de las bridas en el cono de descarga de la turbina de una de las unidades de 90 MW de la Central Hidroeléctrica de Salvajina. Como uno de los antecedentes la UIM de UMIVALLE había previamente diagnosticado el fenómeno de fatiga como responsable de la falla y el trabajo del GIMI se orientó a evaluar las posibles causas de falla, utilizando diferentes técnicas experimentales para evaluación de componentes mecánicos, y a diagnosticar y formular soluciones a los factores que concurren en un fenómeno de esta índole. En este artículo se presenta el análisis teórico y las diferentes técnicas experimentales empleadas, resultados, conclusiones y recomendaciones.

\section{PLANTEAMIENTO DEL PROBLEMA}

La unidad estudiada ha presentado en forma repetida la falla por fatiga de varios tornillos de unión de las bridas del cono de descarga. El cono original (Figura 1) había sido intercambiado con el de otra de las unidades y al parecer este hecho coincidió con el inicio del problema. El sistema de fijación fue planteado inicialmente con roscas en las bridas de norma M-.30 ×3.5 y por el deterioro gradual se eliminaron las roscas de la brida inferior quedando los tornillos pasantes. La unidad fue sometida a reparación por problemas del devanado estatórico y se aprovechó para sustituir los dos segmentos del cono de descarga por dos segmentos nuevos. Las nuevas piezas fueron replanteadas con refuerzos o nervaduras adicionales para rigidizarlas y con asientos para la ubicación de gatos mecánicos para transferir carga a la obra civil (Figura 2).

Por aspectos eminentemente hidráulicos, los niveles de vibración en los conos se elevan significativamente bajo condiciones de carga parcial, por ejemplo con $60 \mathrm{MW}$. 


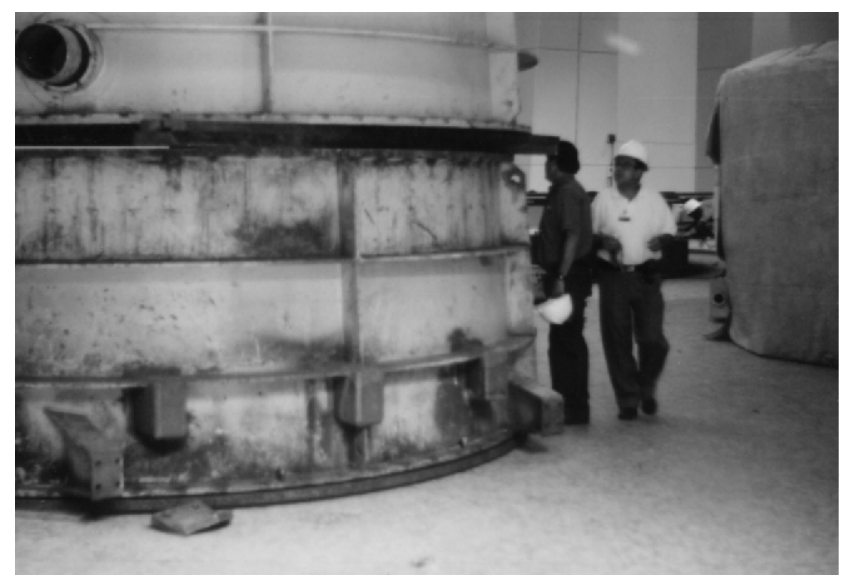

Figura 1. Conos Originales

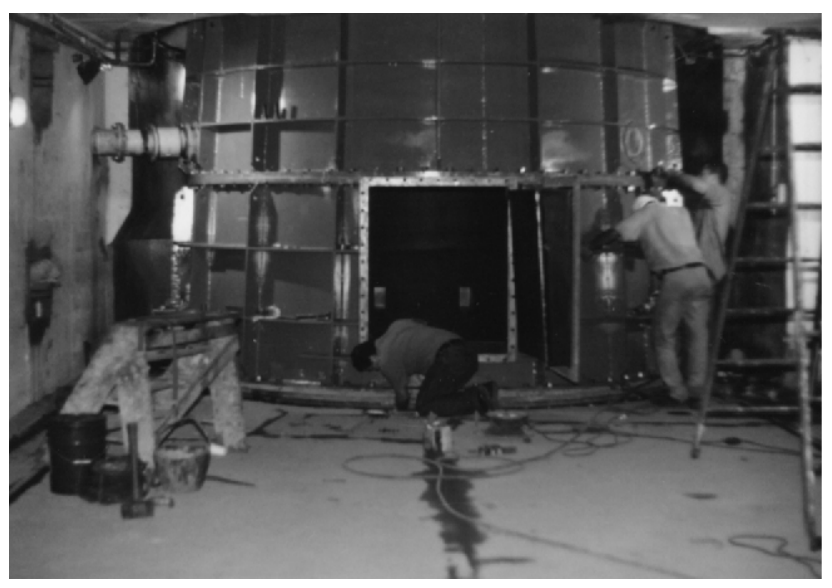

Figura 2. Conos Nuevos rigizados

En el caso de estudio, como los tornillos están sometidos a cargas variables, es conveniente realizar un análisis elástico que incluya el valor de la precarga. Este valor en todos los casos es definido y enunciado por el fabricante, en este caso Toshiba. Dado que el valor del par de apriete inicial y por ende el de la fuerza de precarga han sido cambiados por el usuario (aumentados), conviene revisar los cálculos con un enfoque general que permita ver las implicaciones de ese incremento en la vida en fatiga de los tornillos. Para sacar conclusiones sobre las causas de falla, además de realizar un análisis sobre el comportamiento elástico de la unión y la influencia del par de apriete y la precarga en éste, se efectuó una evaluación experimental sobre los factores más relevantes. Estos son: Propiedades mecánicas de material, metrología y análisis experimental de esfuerzos en los tornillos.

\section{EVALUACIÓN EXPERIMENTAL}

\subsection{Determinación de las Propiedades Mecánicas del Material de los Tornillos}

La propiedad mecánica más importante asociada con sujetadores roscados es la resistencia en tensión, es la guía para el usuario de éstos respecto a la resistencia y a la capacidad del sujetador para soportar carga. En el caso que nos ocupamos se realizaron ensayos de tracción a probetas de diferentes materiales utilizados en la elaboración de los tornillos.

Los datos de las probetas, así como los resultados obtenidos son mostrados en la Tabla Mo. 1

\begin{tabular}{|l|c|c|c|c|}
\hline \multirow{2}{*}{$\begin{array}{c}\text { Identificación del } \\
\text { material y/o perno }\end{array}$} & \multirow{2}{*}{$\begin{array}{c}\text { Diámetro Sección } \\
\text { Transversal }(\mathrm{mm})\end{array}$} & $\begin{array}{c}\text { Fuerza Máxima } \\
\text { (Mewton) }\end{array}$ & \multicolumn{2}{|c|}{ Resistencia a la Tracción } \\
\cline { 4 - 5 } & & Mps & p.5.i. \\
\hline Acero Inoxidable BP42 & 6.85 & 34790 & 944 & 136880 \\
\hline Acero Inoxidable 430 & 6.75 & 19600 & 548 & 79460 \\
\hline Mitsubishi & 7.00 & 26166 & 680 & 98600 \\
\hline F11 & 6.90 & 19992 & 535 & 77575 \\
\hline
\end{tabular}

Tabla Mo. 1: Resistencia en Tracción de Probetas obtenidos a partir de Pernos Utilizados

\subsection{Metrología de los Tornillos}

Se efectuaron mediciones en varios de los tornillos que habían sido utilizados anteriormente - estaban siendo utilizados (Figura 3). Para realizar éstas se utilizaron los siguientes equipos: Mikon Profile Proyector Model 6C y Gran Medidor de Herramientas.

Los resultados de estas mediciones están dados en la Tabla Mo 2

\begin{tabular}{|c|c|c|c|c|c|c|c|}
\hline $\begin{array}{c}\text { Mo. } \\
\text { Tornillo }\end{array}$ & \multicolumn{2}{|c|}{$\begin{array}{c}\text { DIÁMETRO } \\
(\mathrm{mm} .)\end{array}$} & \multirow{2}{*}{$\begin{array}{c}\text { ÁNGULO } \\
\text { DE PERFIL }\end{array}$} & $\begin{array}{c}\text { PASO } \\
(\mathrm{mm} .)\end{array}$ & $\begin{array}{c}\text { PROFUMD. } \\
(\mathrm{mm} .)\end{array}$ & $\begin{array}{c}\text { TIPO DE } \\
\text { ROSCA }\end{array}$ & $\begin{array}{c}\text { FILETE } \\
\text { FIGURA } \\
\text { Mo. }\end{array}$ \\
\hline 1 & 29.728 & 25.149 & $29^{\circ} 25^{\prime}$ & 2.286 & 3.588 & Whitworth & 6 \\
\hline 2 & 29.690 & 25.183 & $23^{\circ} 10^{\prime}$ & 2.254 & 3.916 & Whitworth & 7 \\
\hline 3 & 29.494 & 25.324 & $29^{\circ} 5^{\prime}$ & 2.085 & 3.464 & Whitworth & 8 \\
\hline 4 & 29.570 & 25.086 & $31^{\circ} 13^{\prime}$ & 2.242 & 3.454 & Hibrido Whit.-Met. & 10 \\
\hline 5 & 29.806 & 25.244 & $23^{\circ} 34^{\prime}$ & 2.281 & 3.491 & Whit. Modificado & 9 \\
\hline 6 & 31.773 & 27.480 & $30^{\circ} 51^{\prime}$ & 2.147 & 3.493 & Whit. Modificado & 11 \\
\hline 7 & 29.972 & 25.253 & $30^{\circ} 6^{\prime}$ & 2.360 & 3.524 & Hibrido Whit.-Met. & 12 \\
\hline
\end{tabular}

Tabla Mo. 2: Resultados de Mediciones efectuados a Tornillos 


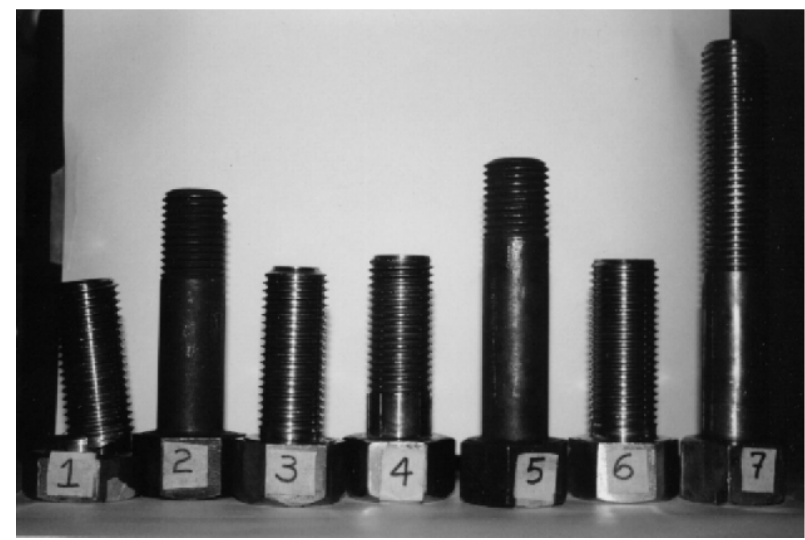

Figura 3. Tornillos a los cuales se efectuaron Mediciones

Desde el punto de vista dimensional se considera que los tornillos tienen las medidas de diámetro y profundidad de la rosca aproximadas a las teóricas (diámetro exterior de $30 \mathrm{~mm}$ y profundidad de rosca de $2,273 \mathrm{~mm}$.)

Aunque las roscas (Figura 4) no presentan una uniformidad en cuanto a su tipo, se considera que la falla de los tornillos no es ocasionada por el tipo - acabado de la rosca. Sin embargo, es recomendable utilizar un solo tipo de rosca y realizar un control dimensional completo, tanto al tornillo como a la tuerca. En conclusión se encuentra una calidad no uniforme en el suministro (fabricación) de los tornillos estudiados.

\subsection{Análisis Experimental de Esfuerzos}

Con el objeto de reunir datos acerca del comportamiento de las uniones por tornillo se diseñaron y construyeron sensores a partir de tornillos suministrados por EPSA (Figura 5).

Estos elementos fueron instrumentados con deformímetros eléctricos (straín gages) para determinar la fuerza axial en diferentes posiciones de las bridas y en uno de los casos se colocó una roseta de tres strain gages arreglados a 0, 45 y 90 grados (Figura 6). Con esta última es posible detectar la dirección y magnitud de los esfuerzos principales los cuales pueden informar sobre la existencia e importancia de los posibles momentos flectores presentes en los tornillos de las bridas inferiores (en las que emplean sectores o cuñas de ajuste).

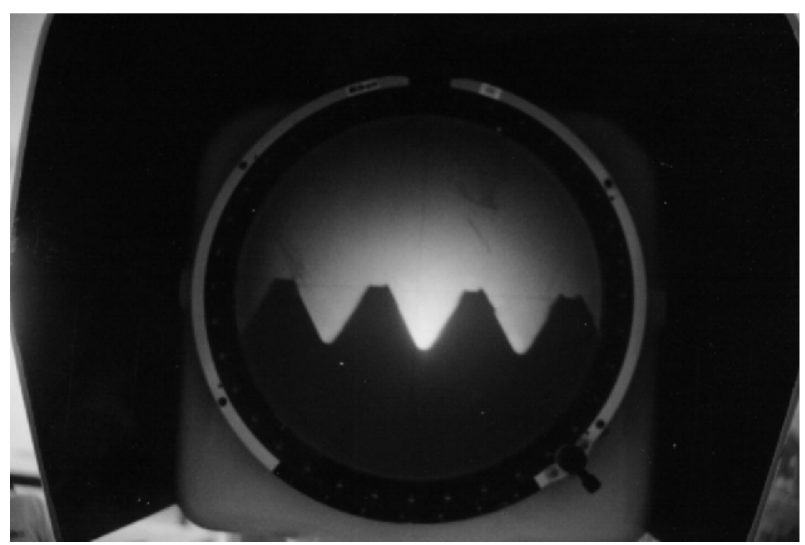

Figura 4. Filete del Tornillo Mo. 7

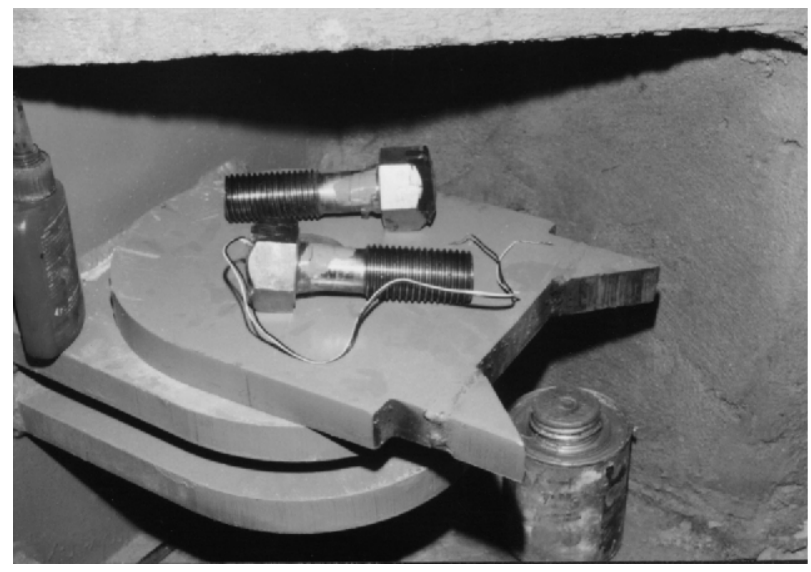

Figura 5. Sensores construidos a partir de Tornillos

Como trabajo adicional se instaló una roseta similar a la anterior en el cuerpo del cono inferior con el objeto de apreciar la magnitud de los esfuerzos adicionales producidos al entrar en operación la unidad. Esta última roseta se instaló después del torqueo final de las uniones y en principio sólo muestra la carga adicional a partir de allí. Para tomar las lecturas de las deformaciones se utilizó el siguiente equipo.

Indicador de Deformaciones, Modelo P-3500, Instruments Division, Measurements Group; 
Unidad de Conexión y Balanceo, Modelo SB-10, Instruments Division, Meaurements Group.

Se efectuaron lecturas de deformaciones en cuatro ocasiones desde el momento del apriete inicial hasta aproximadamente un mes después. El par de apriete inicial fue el producido cuando el indicador de presión de la bomba que acciona el elemento que produce el apriete indicaba aproximadamente 3.000 psi.

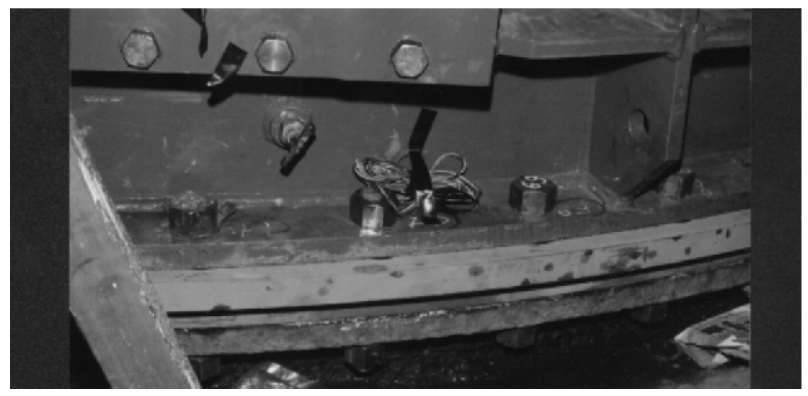

Figura 6. Sensor con roseta utilizado en Brida Inferior

A partir de las deformaciones registradas se calculan los esfuerzos. En la Tabla Mo. 3 se presentan los esfuerzos axiales en los tornillos en los cuales estaban colocados sensores de este tipo.

En la Tabla Mo. 4 se presentan los esfuerzos principales y la dirección de estos (con respecto a la línea vertical) en los puntos donde estaban colocados sensores tipo roseta.

\begin{tabular}{|c|c|c|c|c|}
\hline $\begin{array}{c}\text { POSICIÓM Y Mo. } \\
\text { DEL TORMILLO }\end{array}$ & $\begin{array}{l}\text { DESPUÉS DEL } \\
\text { APRIETE IMICIAL } \\
\text { (Dic. 19/1999) }\end{array}$ & Dic. 22/1999 & Ene. 4/2000 & Ene. 24/2000 \\
\hline Brida Superior Mo. 25 & 54960 & 51780 & 51300 & 46260 \\
\hline Brida Superior Mo. 72 & 65070 & 61260 & 59700 & 57990 \\
\hline Brida Inferior Mo. 46 & 94800 & 79200 & 73800 & 69780 \\
\hline Brida Inferior Mo. 22 & 79920 & 77100 & 76440 & 75900 \\
\hline
\end{tabular}

Tabla Mo. 3: Esfuerzos Axiales (en p.s.i.) En Tornillos

\begin{tabular}{|c|c|c|c|c|c|}
\hline ELEMENTO & \begin{tabular}{c|} 
ESFUERZOS \\
PRIMCIPALES (p.S.I.) \\
Y DIRECCIOMES (")
\end{tabular} & $\begin{array}{l}\text { DESPUÉS DE } \\
\text { APRIETE }\end{array}$ & Dic. 22/1999 & Ene. 4/2000 & Ene. 24/2000 \\
\hline \multirow{4}{*}{$\begin{array}{c}\text { ROSETA } \\
\text { EM TORMILLO } \\
\text { MO. } 70 \\
\text { BRIDA INFERIOR }\end{array}$} & Esfuerzo Principal 1 & 11242 & 17248 & 13163 & 23847 \\
\hline & Dirección Esf. Prin. 1 & -243 & -143 & -36.8 & -32.3 \\
\hline & Esfuerzo Principal 2 & -15637 & -6748 & -275 & 3375 \\
\hline & Dirección Esf. Prin. 2 & 65.7 & 75.7 & 53.2 & 57.7 \\
\hline \multirow{4}{*}{$\begin{array}{c}\text { ROSETA } \\
\text { EN CUERPO } \\
\text { COMO IMFERIOR }\end{array}$} & Esfuerzo Principal 1 & & 2925 & 14875 & 19611 \\
\hline & Dirección Esf. Prin. 1 & & -22.5 & -4 & -4 \\
\hline & Esfuerzo Principal 2 & & -5355 & -5355 & 11031 \\
\hline & Dirección Esf. Prin. 2 & & 67.5 & 86 & 86 \\
\hline
\end{tabular}

Tabla Mo. 4: Esfuerzos Principales (en p.s.i.) y su Dirección en Rosetas

De acuerdo a los resultados presentados en las Tablas Mos. 3 y 4, se construyeron gráficos esfuerzo axial vs. Tiempo (Figura 7) para cada tornillo instrumentado con este tipo de sensor $y$ esfuerzos principales vs. tiempo para cada roseta (Figura 8).

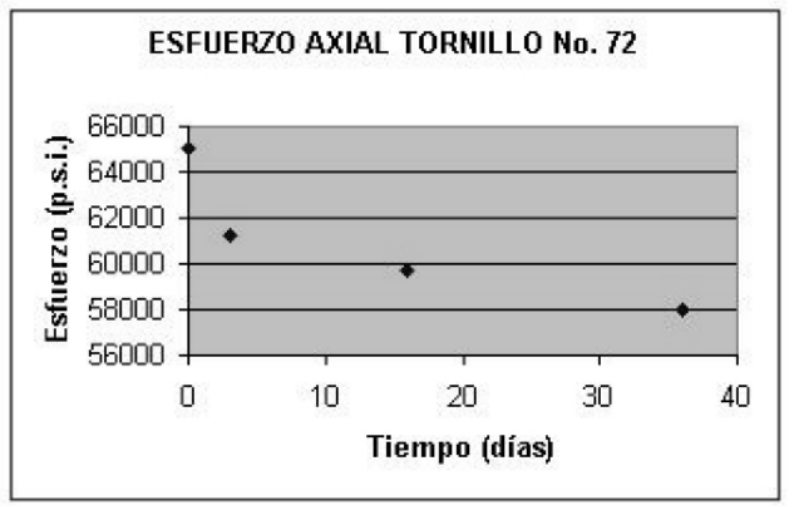

Figura 7. Esfuerzo Axial vs. Tiempo en Tornillo Mo. 72 Brida Superior

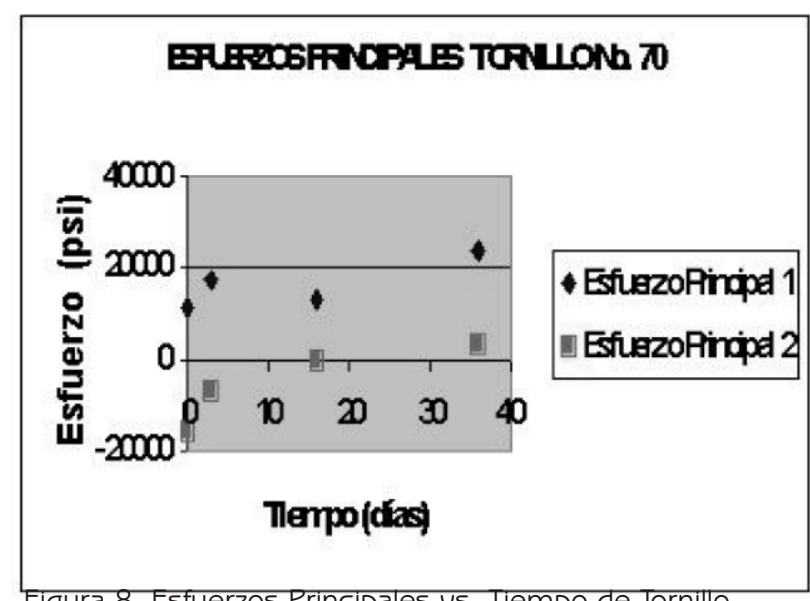

Mo. 70 en Brida Inferior 


\section{ANÁLISIS ELÁSTICO. CONSIDERACIONES SOBRE EL PAR DE APRIETE Y LA PRECARGA}

El continuo énfasis en alcanzar y mantener control apropiado en la precarga de los tornillos nos lleva a una situación dramática: la persona que tiene la mayor influencia en la integridad estructural de la unión ensamblada es aquella que tiene la llave para apretar (las tuercas de los tornillos). Si el par torsor inicial es muy alto, hay peligro de falla en la instalación por daño de la rosca o rotura del tornillo, o haciendo que éste fluya excesivamente. Si el perno es torqueado muy bajo, una baja precarga se inducirá en el ensamble, provocando posiblemente falla por fatiga y/o vibraciones. Para cada sistema de sujetador roscado, hay una óptima precarga, la cual es obtenida por el torqueo apropiado de la combinación perno-tuerca.

La cantidad de apriete normalmente requerida para elongar un perno e inducir una precarga en tensión deseada en el sistema de sujeción se identifica generalmente como la relación torquetensión. Esta relación es a menudo ignorada. A menos que la relación torque-tensión sea aplicada con exactitud, hay un inminente peligro de perder todas las ventajas obtenidas por un buen diseño de la unión.

Los sujetadores roscados tales como pernos y tornillos responden de forma diferente a la aplicación de una carga axial en tensión que a un estiramiento por aplicación de torque. Muchas recomendaciones de diseño se formulan para torquear los sujetadores hasta que desarrollen una precarga en tensión de 60 a $80 \%$ de la resistencia estática en tensión. El torquear en exceso puede resultar en excesiva elongación del perno y por consiguiente en la posible falla de ésta.

El problema de torquear para alcanzar la precarga de tensión involucra el tema crítico de los coeficientes de fricción del sistema de sujeción. Los investigadores en este tema han establecido que el $90 \%$ del par torsor aplicado es usado para vencer la fricción en los tornillos roscados, con los puntos de mayor fricción bajo la cabeza del tornillo, bajo la tuerca y en la rosca. Este extenso trabajo ha indicado que la siguiente fórmula puede ser usada para estimar la precarga en tensión ocasionada por el torqueo:

$$
T=K D P
$$

Donde:

$T=$ par torsor de instalación. Ibf - pulgadas

$K=$ Coeficiente de torque

$\mathrm{D}=$ Diámetro nominal, pulgadas

$\mathrm{P}=$ Precarga, $\mathrm{Ibf}$

De la fórmula se puede ver que la principal variable es el coeficiente de torque $K$. Este coeficiente varía con diferentes acabados, recubrimientos electrolíticos y lubricantes normalmente encontrados en tornillos normalizados.

El mayor énfasis para mantener el control de la precarga está en controlar el par torsor con exactitud. Para aplicaciones críticas, el uso de una llave de torque es una necesidad. Aún con una llave de torque, se puede observar una amplia gama de valores de precarga.

Un coeficiente de torque de 0,20 es adecuado para tornillos no lubricados, mientras que un coeficiente de 0,15 es recomendado para acabados con recubrimientos electrolíticos. El uso de lubricantes adicionales como aceite, grafito, cera, etc., puede variar el factor $K$ desde 0,07 hasta 0,30. Para aplicaciones específicas de diseño con requerimientos de control exacto de precarga, puede ser necesario ensayar y evaluar los pernos y tuercas especificados para el diseño para definir los valores necesarios del torque de instalación. 
Para realizar un análisis elástico de la junta se tomará como límite de fluencia, $S_{y^{\prime}}$ del material empleado 70.000 p.s.i. Aunque se dispone del valor de la resistencia última de algunos de los materiales utilizados en los tornillos (Tabla Mo. 1), la utilización de diferentes materiales en la elaboración de éstos no nos permite generalizar. La recomendación del fabricante, en este caso Toshiba, es aplicar un par de apriete entre 383 y 520 Ibf. pie (para un tornillo de la calidad del Mitsubishi seguramente).

Si tomamos $K=0.15$ y diámetro nominal de 1.18 pulgadas (30 mm.) obtendríamos que, de acuerdo a esta recomendación, la precarga $\mathrm{P}$ tendría un valor entre 25966 y 35254 Ibf. Tomando como diámetro de raíz efectivo 1 pulgada, el esfuerzo producido por esta precarga estará en un rango entre 33000 y 45000 psi. Si se acepta $S_{y}=70000$ psi (para un acero 420) la precarga estaría entre 0.475 y y $0.645_{y}$, lo cual se considera adecuado.

Si el límite de fluencia es 50000 psi este rango estará entre 0.665 y y 0.95 y lo cual sería alto (precarga alta) y si el límite de fluencia es 100000 psi el rango estará entre 0.335 y y $0.45 \mathrm{~S}_{\text {y }}$ (precarga baja).

Se evalúa el efecto de aplicar precarga baja, adecuada y alta.

El análisis elástico se realiza con las siguientes consideraciones:

* El módulo elástico de los tornillos es $28.5\left(10^{6}\right)$ p.s.i., mientras que el de las partes (bridas, anillo y cuña) es $30\left(10^{6}\right)$ p.s.i.

* El límite de fatiga para el acero se supone de 30000 p.s.i., sin incluir factor por concentración de esfuerzos.

* El factor de concentración de esfuerzos $K_{\mathrm{f}}$ para la rosca es de 3.8 (rosca torneada).

* El diámetro nominal de los tornillos es 1.18 pulgadas $(30 \mathrm{~mm}$.) y por lo tanto su área transversal $A_{b}=(\pi / 4)(1.18)^{2}=1.1 \mathrm{pg}^{2}$.

* Como área efectiva de las partes se toma $A_{p}=(\pi / 4)\left((3 d)^{2}-d^{2}\right)=8.75 p^{2}$.

Las constantes de elasticidad serán por lo tanto,

$K_{b}=\left(A_{b} E_{b}\right) / L_{b}=(1.1)\left(28.5 * 10^{6}\right) / 6=5.22 * 10^{6}$ Ibf./ pulg.

$K_{p}=\left(A_{p} E_{p}\right) / L_{p}=(8.75)\left(30 * 10^{6}\right) / 6=43.7^{*} 10^{6}$ lbf./ pulg.

Al aplicar sobre el tornillo una fuerza externa $F_{e}$ se tendrá que:

$F_{b}=P+\left(K_{b} /\left(K_{b}+K_{p}\right)\right) F_{e}$

Si esta fuerza externa $F_{\text {e }}$ fluctúa entre un valor mínimo y un máximo, obtenemos una fuerza externa media y una fuerza externa alterna, cuyos valores están dados por

$F_{b m}=P+\left(K_{b} /\left(K_{b}+K_{p}\right)\right) F_{e m}=P+0.11 F_{e m}$

$F_{\text {ba }}=\left(K_{b} /\left(K_{b}+K_{p}\right)\right) F_{\text {ea }}=0.11 F_{\text {ea }}$

Los esfuerzos medio $\mathrm{s}_{\mathrm{m}}$ y alterno $\mathrm{s}_{\mathrm{a}}$ correspondientes serán

$\sigma_{\mathrm{m}}=\mathrm{F}_{\mathrm{bm}} / \mathrm{A}=\mathrm{F}_{\mathrm{bm}} / 1 \mathrm{pg}^{2}$

$\sigma_{\mathrm{a}}=\mathrm{K}_{\mathrm{f}} \mathrm{F}_{\mathrm{ba}} / A=K_{\mathrm{f}} F_{\mathrm{ba}} / 1 \mathrm{pg}^{2}$

Como ilustración, consideremos que la fuerza externa fluctúa entre 500 lbf y 13500 lbf en cada tornillo. En este caso $F_{\text {em }}=7000$ lbf y $F_{\text {ea }}=6500$ Ibf. Por lo tanto

$\mathrm{F}_{\mathrm{bm}}=\mathrm{P}+0.11 \mathrm{~F}_{\mathrm{em}}=\mathrm{P}+770 \mathrm{lbf}$

$\mathrm{F}_{\mathrm{ba}}=0.11 \mathrm{~F}_{\mathrm{ea}}=715 \mathrm{lbf}$.

En la Tabla Mo. 5 se dan los valores de $\sigma_{\mathrm{m}}$ y $\sigma_{\mathrm{a}}$ para diferentes valores de fuerza de precarga y en la Figura 8 se muestra un diagrama $\sigma_{\mathrm{m}} \mathrm{vs}$. $\sigma_{\mathrm{a}}$ donde se comparan las diferentes situaciones de precarga. 


\begin{tabular}{|l|c|c|c|}
\hline Precarga, $\mathrm{P}(\mathrm{Ibf})$ & $\sigma \mathrm{m}$ (PSi) & $\sigma_{\text {a }}(\mathrm{PSI})$ & Ubicación en Figura 8 \\
\hline Baja, $\mathrm{P}=0$ & 7000 & 24700 & Punto D \\
\hline Adecuada, $\mathrm{P}=32000$ & 32770 & 2717 & Punto a \\
\hline Alta, $\mathrm{P}=60000$ & 60770 & 2717 & Punto C \\
\hline
\end{tabular}

Tabla Mo. 5: Esfuerzos Medios y Alternos para Diferentes Precargas

La Figura 9 nos muestra muy claramente que tanto la ausencia (punto b) como el exceso (punto c) de precarga pueden sacar la unión de las condiciones seguras.

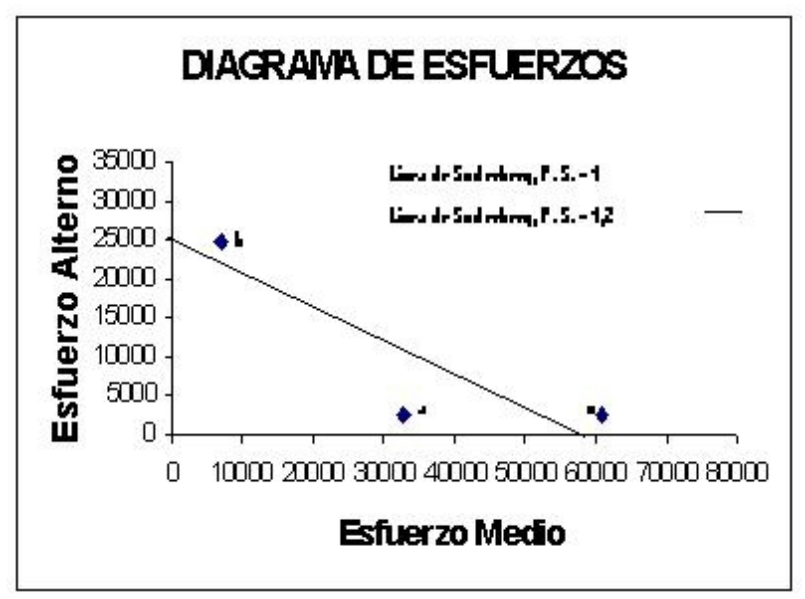

Figura 9. Diagrama de Esfuerzos para juntas sometidas a cargas fluctuantes

\section{CONCLUSIONES $Y$ RECOMENDACIONES}

1. Los resultados de resistencia en tracción de probetas obtenidas de tornillos de diferentes materiales, utilizados en la construcción de estos, muestran diferencias bastante grandes. Es importante notar que aunque los ensayos efectuados no son normalizados, el valor de resistencia obtenido para el acero BP 42 está muy cercano al valor esperado, de acuerdo a la información técnica sobre este acero presentada por uno de los fabricantes de tornillos.

2. El análisis de geometría de los tornillos indica una calidad no uniforme en la fabricación de los mismos.
3. Los esfuerzos que se presentan en los tornillos que tenían colocados sensores axiales (Tabla Mo. 4) nos muestran valores que están en un rango bastante amplio, lo cual permite concluir que el control del par de apriete por medio del instrumento de presión de la bomba es inadecuado, pues no permite aplicar con una buena aproximación el par de apriete requerido.

4. Los esfuerzos a los cuales son sometidos algunos tornillos al apretar la tuerca son muy altos. En el caso de emplear tornillos de baja resistencia. aproximadamente 60000 p.s.i. de límite de fluencia o 80000 p.s.i. de resistencia último se corre el riesgo que fallen desde el apriete.

5. Debido a las dimensiones y geometría de las piezas unidas y dependiendo del proceso de torqueo, aún en condiciones estáticas, es posible que durante la aplicación de torque a los tornillos inferiores, algunos tornillos de la junta superior pueden alcanzar valores importantes de carga media. Un valor de esfuerzo estático excesivo puede originar flujo plástico en las raíces de los filetes y propiciar un pronto inicio de la falla por fatiga.

6. Por otro lado, no es posible garantizar la homogeneidad del comportamiento de los tornillos bajo carga dinámica particularmente si se presenta aflojamiento en forma parcial y localizada. Al observar las Figuras 7 y 8 notamos que los esfuerzos axiales disminuyen en el tiempo, mientras que los esfuerzos principales aumentan. Esto nos indica que los tornillos se van aflojando, pero a medida que esto sucede entra a actuar una componente de momento flector, responsable del aumento de los esfuerzos principales. 
7. El hecho que se cambie a materiales más resistentes para tornillos, no necesariamente obliga a subir el torque de precarga y puede considerarse como un incremento en el factor de seguridad de la junta.

8. Se recomienda que se reduzca el torque de apriete a valores en el rango sugerido por Toshiba. basado en el análisis anterior que evidencia el riesgo de falla en las uniones evaluadas.

9. Es urgente someter el suministro de tornillos a un control tanto de material como de geometría.

10.La solución al problema es integral: material, geometría y precarga.

\section{BIBLIOGRAFÍA}

Beckwith T.G. y Marangoni R.D.; "Mechanical Measurements", Addisson Wesley y Publishing Co, Inc., Canada 1990.

Doebelin E.O.; "Engineering Experimentation", MC Graw Hill, Co., Singapur 1995.

Dally J.W. y Riley W.F.; "Experimental Stress Analysis", Mc Graw Hill, Co., Mew York 1978.

Morton R.L.; "Diseño de Máquinas", Prentice HallPearson, Mexico 1999.

Spotts M.F. y Shoup T.E., "Elementos de Máquinas", Prentice Hall, Mexico 1998.

Burr A. H. Y Cheatham J.B. "Mechanical Analysis Design", Prentice Hall, Mew Jersey 1995.

Parmley R.O., "Standard Hhandbook of Fastening and Joining, Mc Graw Hill Co, Mew York 1977.

Juvinal R.C. "Fundamentals of Machine Components Design", John Wiley y Sons, Mew York 1983. 